\title{
Evaluation of Four Methods for Detecting the Beta-lactamase Activity in Neisseria gonorrhoeae Isolated in Cuba
}

\author{
Rafael Llanes/+ , Miriam González/ ${ }^{++}$, Isabel Martínez, Jorge Sosa, Daymi Guzmán, \\ Oderay Gutiérrez, Alina Llop, Lizet Sánchez*
}

\author{
Departmento de Bacteriología *Departmento de Informática, Instituto de Medicina Tropical Pedro Kourí, Apdo Postal 601, \\ Marianao 13, Ciudad de La Habana, Cuba
}

Four methods (chromogenic, acidimetric, inhibition, and iodometric) for demonstration of the beta-lactamase production by 70 isolates of Neisseria gonorrhoeae, were evaluated in Cuba. There was $100 \%$ correlation between all beta-lactamase methods and the standardized penicillin dilution susceptibility test for penicillinase-nonproducing N. gonorrhoeae. For penicillinase-producing N. gonorrhoeae strains, there was a perfect correlation between the chromogenic method and penicillin susceptibility testing, but one and two strains failed to give a positive result for beta-lactamase with the inhibition/acidimetric and the iodometric methods, respectively. There was a high concordance between the chromogenic method, considered as gold standard and the rest of penicillinase tests evaluated: Kappa Index (KI) $=0.98$ for inhibition/acidimetric methods and KI $=0.97$ for the iodometric method. The four methods evaluated were accurate, reproducible, easily readable, economical, and ease to use for screening primary isolates of $\mathrm{N}$. gonorrhoeae in Cuba. We recommended the use of the inhibition method, when testing the penicillinase activity in gonococcal isolates in provincial and municipal reference laboratories.

Key words: Beta-lactamase - Neisseria gonorrhoeae - Cuba

Gonorrhea remains one of the most common sexually transmitted infections (STI) and a global health problem (Mayaud et al. 2002). The problem is enhanced by the developing resistance to antibiotics of Neisseria gonorrhoeae, a result of both the wide dissemination of resistant clones and the emergence of strains with novel mechanisms of resistance (Llanes et al. 2003).

The beta-lactamases are the group of enzymes capable of hydrolyzing the beta-lactam bond of both penicillins and some cephalosporins, thereby causing these antibiotics to become inactive (Nicolas-Chanoine 1996). Several methods are available for detecting the production of penicillinase by bacteria that include: iodometric, acidimetric, chromogenic cephalosporin, inhibition, and reverse passive haemaglutination procedures (Hodge et al. 1978, Oberhofer \& Towle 1982, Livermore 1995).

According to the Cuban Program for Control of STI, penicillin is the drug of choice for treatment of uncomplicated gonorrhea. For those individuals developing therapeutic failures to this antibiotic, the realization of culture

The Cuban Ministry of Public Health has recently changed the treatment for gonorrhea. Now, ciprofloxacin at the dose of 500 $\mathrm{mg}$ orally is the new recommended antimicrobial in Cuba (Ministry of Public Health of Cuba 2002).

${ }^{+}$Corresponding author. Fax: + 537-204.6051. E-mail: llanesrafael2002@yahoo.com

${ }^{++}$Recipient of a Master Degree at the Tropical Medicine Institute Pedro Kourí

Received 5 May 2003

Accepted 13 November 2003 and the study of the beta-lactamase activity of isolates are indicated, at municipal or provincial reference laboratories (Ministerio de Salud Pública de Cuba 1997). However, in recent visits made by the Cuban National Reference Laboratory for Neisseria (NRL), to all provincial and many municipal laboratories, only $8 / 14$ provinces and less than $50 \%$ of municipal laboratories were determining the beta-lactamase activity of gonococcal isolates, due to different reasons, mainly economical: limitation of reagents, poor refrigeration, etc. (NRL, unpublished data, 2002).

The purpose of the present investigation was to evaluate four methods for detecting the penicillinase activity of $N$. gonorrhoeae strains in Cuba and to compare their suitability for routine use in clinical microbiology laboratories.

Seventy strains of $N$. gonorrhoeae isolated from urogenital samples in both male and female patients with symptomatic gonorrhea, were studied at the NRL, Tropical Medicine Institute Pedro Kouri in Havana, Cuba. These strains were recovered from different Cuban provinces in the period January 1995 to May 1997. The isolates were cultured onto modified Thayer-Martin-agar (Biocen, Cuba) plates and incubated at $35^{\circ} \mathrm{C}$ in a $\mathrm{CO}_{2}$ atmosphere, for 24 to $48 \mathrm{~h}$. Colonies of $N$. gonorrhoeae were identified by conventional methodology (Llanes et al. 2001).

Four methods for determination of beta-lactamase were used: (1) inhibition method, according to the methodology of Palmer et al. (2000), using chocolate agar plates containing $1 \mathrm{mg} / \mathrm{l}$ of ampicillin, and the Oxford strain of Staphylococcus aureus (NTCC 6571) as a biological indicator; (2) iodometric method in tube (Thornsberry 1980); (3) acidimetric method of Thornsberry and Kirven (1974), using paper disc impregnated with a solution of 
penicillin and phenol red as a $\mathrm{pH}$ indicator; (4) chromogenic cephalosporin method, employing a commercial reagent (Nitrocefin, Oxoid), prepared as liquid solution (Llanes et al. 2000).

Two gonococcal strains, WHO-E and WHO-A, and two strains of S. aureus, ATCC 29213 and ATCC 25923, were used as positive and negative controls for betalactamase activity, respectively.

Parallel agar dilution penicillin susceptibility tests were performed on GC agar base (Biocen) plus $1 \%$ Vitox supplement, according to the recommendations of the National Committee for Clinical Laboratory Standards (NCCLS 2000).

Using the chromogenic cephalosporin method as gold standard for beta-lactamase test (Livermore 1995), we determined the concordance with the rest of methods studied, by using the Kappa Index (KI) as a comparison test; $\mathrm{P}<0.05$ was accepted as statistical significant. The sensitivity, specificity, predictive values and confidence intervals were calculated (Fleiss 1981). The four methods were also compared on the basis of their readability, reproducibility, speed of performance, ease of preparation of reagents, and relative cost.

In the present study, from the 70 strains of $N$. gonorrhoeae investigated, 39 were susceptible or displaying intermediate susceptibility to penicillin: minimal inhibitory concentration (MIC $\leq 0.015-1 \mathrm{~g} / \mathrm{l}$ ), and 31 showed a high resistance to this antibiotic (MIC $\geq 16 \mathrm{~g} / \mathrm{l}$ ) (Llanes et al. 2003). All those 39 strains showed the absence of penicillinase activity by any of the methods used. Thus, there was $100 \%$ correlation between susceptibility results and beta-lactamase tests for $N$. gonorrhoeae penicillinasenon-producing (non-PPNG) strains. For penicillinaseproducing $N$. gonorrhoeae (PPNG) strains, there was a perfect correlation between susceptibility results and betalactamase test by the chromogenic method (31/31 strains). However, by the inhibition/acidimetric and the iodometric methods, one and two strains, respectively, appeared to be highly resistant to penicillin (MIC $\geq 16 \mathrm{~g} / \mathrm{l}$ ), but failed to give positive results for penicillinase activity (Table I).

There was a high concordance (KI) between the different beta-lactamase methods used and the chromogenic method. For the iodometric method KI $=0.97$, and for inhibition/acidometric KI $=0.98(\mathrm{p}<0.0001)$.

Sng et al. (1981) achieved a complete agreement between the acidimetric, chromogenic, and iodometric methods in 300 strains of $N$. gonorrhoeae isolated in Singapore. Meanwhile, Oberhofer and Towle (1982) and
Thornsberry (1980) observed comparable results using the acidimetric/iodometric and acidimetric/chromogenic methods, in gonococci, respectively. Comparison of inhibition method with other beta-lactamase tests has given identical results (Jorgensen et al. 1977).

In our study when estimating the sensitivity and specificity of the penicillinase methods, the following results were observed: a sensitivity of $96.8 \%$ for acidimetric and inhibition methods, and of $93.5 \%$ for iodometric one. The specificity for all methods was $100 \%$ (Table II). Similar results have been reported by Hodge et al. (1978), Sng et al. (1981), and Montgomery et al. (1979). All beta-lactamase, methods showed a high re-

TABLE II

Sensitivity, specificity, positive, and negative predictive values for beta-lactamase methods evaluated

\begin{tabular}{lcccc}
\hline & $\begin{array}{c}\text { Iodometric } \\
(\%)\end{array}$ & CI (\%) & $\begin{array}{c}\text { Acidimetric and } \\
\text { inhibition }(\%)\end{array}$ & CI (\%) \\
\hline Sensitivity & 93.5 & $80.2-96.6$ & 96.8 & $84.5-99.3$ \\
Specificity & 100 & $91.3-100$ & 100 & $91.3-100$ \\
PPV & 100 & $88.6-100$ & 100 & $88.9-100$ \\
NPV & 96.1 & $84.6-99$ & 97.6 & $87.7-99.8$
\end{tabular}

CI: confidence interval; PPV: positive predictive value; NPV: negative predictive value

producibility and gave clear readable results. The iodometric, acidimetric, and chromogenic methods produced the most distinctive color changes. The inhibition method was particularly good respect to readability.

For the inhibition method it is necessary to make a subculture of the organism: $18-20 \mathrm{~h}$, which implies a delay in the determination of penicillinase activity. Strains required less than $1 \mathrm{~min}$ with the chromogenic method and less than $30 \mathrm{~min}$ with the iodometric and acidimetric tests.

For all methods it is necessary some previous preparation of reagents, however that preparation is not at all complex or time consuming. The majority of stock reagents, once prepared, can be stored for some time. With the exception of the chromogenic cephalosporin method, all the materials needed are inexpensive and available.

TABLE I

Correlation between beta-lactamase methods and penicillin susceptibility in 70 strains of Neisseria gonorrhoeae isolated in Cuba

\begin{tabular}{|c|c|c|c|c|c|c|c|c|c|}
\hline \multirow{2}{*}{$\begin{array}{l}\text { Antibiotic susceptibility } \\
\text { Interpretive category }\end{array}$} & \multirow[b]{2}{*}{ Nr tested } & \multicolumn{2}{|c|}{ Iodometric } & \multicolumn{2}{|c|}{ Acidimetric } & \multicolumn{2}{|c|}{ Inhibition } & \multicolumn{2}{|c|}{ Chromogenic } \\
\hline & & Pos & Neg & Pos & Neg & Pos & Neg & Pos & Neg \\
\hline Resistant (MIC $\geq 16 \mathrm{~g} / \mathrm{l})$ & 31 & 29 & 2 & 30 & 1 & 30 & 1 & 31 & 0 \\
\hline $\begin{array}{l}\text { Susceptible or Intermediate } \\
(\mathrm{MIC} \leq 0.015-1 \mathrm{~g} / 1)\end{array}$ & 39 & 39 & 0 & 39 & 0 & 39 & 0 & 39 & 0 \\
\hline
\end{tabular}


Each of the four beta-lactamase tests evaluated in the present study has advantages and disadvantages. However all proved to be reliable, accurate, and easy to use for screening primary isolates of $N$. gonorrhoeae in Cuba.

We feel that the inhibition method may be a suitable alternative for laboratories which lack facilities for reagents preparation and storage, because of the following reasons: (1) using a standard antibiotic sensitivity testing method and readily available penicillin solution; (2) the preparation of plates is simple, and when penicillin has been added to the poured plates are also relatively stable and proved to be reliable for up to 2 weeks at $4^{\circ} \mathrm{C}$; (3) as many as 28 cultures can be tested on a simple $90 \mathrm{~mm}$ Petri dish plate, being an economical technique in this age of economical shortages; (4) maintaining sensitive and control staphylococci' strains on a slant or plate over a long period of time is quite easy; (5) permitting to observe any existing contamination.

After the completion of this investigation the inhibition method will be adopted in the NRL and in provincial and municipal reference laboratories in Cuba, for investigating the penicillinase-production in $N$. gonorrhoeae isolates.

\section{ACKNOWLEDGEMENTS}

To Dr Andrew Turner (PHLS Laboratory, Bristol, UK) and to Dr Susana Fiorito (Instituto Malbrán, Buenos Aires, Argentina), for providing control strains. R Llanes thanks the Japanese International Cooperation Agency for provision of literature for manuscript preparation.

\section{REFERENCES}

Fleiss JL 1981. Statistical Methods for Rates and Proportions, John Wiley \& Sons, New York, p. 217-219.

Hodge W, Ciak J, Tramont EC 1978. Simple method for detection of penicillinase-producing $N$. gonorrhoeae. J Clin Microbiol 7: 102-103.

Jorgensen JH, Lee JC, Alexander GA 1977. Rapid penicillinase paper strip test for detection of beta-lactamase-producing Haemophilus influenzae and Neisseria gonorrhoeae. Antimicrob Agents Chemother 11: 1087-1088.
Livermore DM 1995. Beta-lactamases in laboratory and clinical resistance. Clin Microbiol Rev 8: 557-584.

Llanes R, Sosa J, Guzmán D, Llop A, Gutiérrez Y, Ricardo O 2001. N. gonorrhoeae resistant to ciprofloxacin. First report in Cuba. Sex Transm Dis 28: 82-83.

Llanes R, Sosa J, Martínez I 2000. Detection of penicillinaseproducing N. gonorrhoeae in Cuba, 1995-1999. Sex Transm Inf 76: 58-59.

Llanes R, Sosa J, Martínez I, Valdés EA, Palma S, Llop A, Lantero M 2003. Antimicrobial susceptibility of N. gonorrhoeae strains isolated in Cuba. Implications for therapy of gonorrhea. Sex Transm Dis 30: 10-14.

Mayaud P, West B, Lloyd-Evans N, Seck K 2002. GASP-WAR: West African network to tackle gonorrhoea. The Lancet 359: 173.

Ministerio de Salud Pública de Cuba 1997. Programa de Control de la Blenorragia en Cuba.

Montgomery K, Raymond L, Drew WL 1979. Chromogenic cephalosporin spot test to detect beta-lactamase in clinically significant bacteria. J Clin Microbiol 9: 205-207.

NCCLS-National Committee for Clinical Laboratory Standards 2000. Performance Standards for Antimicrobial Susceptibility Testing, M100-S10, NCCLS, Wayne, PA.

Nicolas-Chanoine MH 1996. Impact of B-lactamases on the clinical use of ß-lactams antibiotics. Int J Antimicrob Agents 7: S21-S26.

Oberhofer TR, Towle DW 1982. Evaluation of the rapid strip test for detection of beta-lactamase. J Clin Microbiol 15: 196-199.

Palmer HM, Leeming JP, Turner A 2000. A multiplex polymerase chain reaction to differentiate B-lactamase plasmids of Neisseria gonorrhoeae. J Antimicrob Chemother 45: 777-782.

Sng EH, Yeo KL, Rajan VS 1981. Simple method for detecting penicillinase-producing Neisseria gonorrhoeae and Staphylococcus aureus. Br J Vener Dis 57: 141-142.

Thornsberry C 1980. Detection of beta-lactamase in Neisseria gonorrhoeae and Haemophilus influenzae, Laboratory Training and Consultation Division Services, Centers for Disease Control, p. 1-8.

Thornsberry C, Kirven LA 1974. Ampicillin resistance in Haemophilus influenzae as determined by a rapid test for beta-lactamase production. Antimicrob Agents Chemother 6: 653-654. 